\title{
STABILITY OF ASTEROID MOTIONS
}

\author{
Y. KOZAI \\ National Astronomical Observatory, Mitaka, Tokyo 181, Japan.
}

\begin{abstract}
In this paper the author presents evidences showing that for most of the asteroids the motions are stable in the sense that they never approach major planets very closely and explains about mechanisms to avoid very close approaches by investigating the variations due to the secular perturbations of the eccentricities as functions of the arguments of perihelion, particularly, for asteroids with high eccentricities and inclinations. It is believed that some kinds of dynamical evolution processes have made the asteroid motions stable. The author shows also that there were some kinds of collisions among asteroids in the past which produced families and present distribution of asteroids as there are very faint asteroids only near Kirkwood gaps.
\end{abstract}

\section{Introduction}

Planetary motions are stable in the sense that their semi-major axes do not change very much and their orbital eccentricities and inclinations are kept to be very small according to the secular perturbation theory. By such ways the planets can avoid mutual collisions. The same is true between planets and asteroids with small eccentricities and inclinations and with orbits in the so-called main-belt.

However, there are several exceptional cases, for which the eccentricities and inclinations can change very much, such as those with high eccentricities and inclinations as well as those with resonant mean motions and/or secular motions. In fact for many of the asteroids the eccentricities and the inclinations are not small and there are many asteroids with commensurable mean motions with Jupiter.

One such example is the case of 1-to-1 mean motion resonance(Trojan asteroids) and for them the critical arguments, the differences between the mean longitudes of Jupiter and the asteroids, are librating around $\pm 60^{\circ}$ and their eccentricities are usually small and inclinations are high. That is why they cannot approach Jupiter very closely.

For 4-to-3(Thule) and 3-to-2(Hilda) commensurable cases there are also many asteroids. However, for most of them the critical arguments are librating so that they make opposition with Jupiter only when they are near perihelia. Therefore, they cannot approach very closely to Jupiter, particularly, for asteroids with large eccentricities. There are a few asteroids, for which the arguments of perihelion are not librating for 3-to-2 case. However, for them the eccentricities are usually small and, therefore, even their smallest distances to Jupiter are not so small. There are few asteroids with semi-major axes larger than 3.7AU, for which, of course, the aphelion distances are larger than 3.7AU, except for such commensurable cases.

For other commensurable cases there are few asteroids, namely, Kirkwood gaps exist there. Still asteroids near the gaps have librating critical arguments and mostly high eccentricities and inclinations to avoid very close approach to Jupiter.

For asteroids with resonant secular motions their eccentricities and/or inclinations can vary very much due to the secular perturbations and, therefore, there are possibilities for them to approach very closely to Jupiter when the eccentricities, and, therefore, the aphelion distances become large. However, asteroids do not exist in any secular resonant region. 
If the inclinations are very large, the eccentricities can change very much and even though the present eccentricities are small, they can take large values due to the secular perturbations. Still there are mechanisms for such asteroids to avoid very close approach to Jupiter. In this paper the motions of asteroids with high eccentricities and inclinations are studied and mechanisms to avoid very close approach are explained.

\section{Secular Perturbation Theory}

Secular perturbation theory is formulated by averaging the Hamiltonian with respect to the mean anomalies of the asteroid concerned and the disturbing planets. Then as the mean anomaly does not appear in the Hamiltonian the semi-major axis becomes constant.

When the eccentricity and the inclination are very small, the equations of motion are reduced to the two sets of linear differential equations, one for the eccentricity and the longitude of the perihelion and one for the inclination and the longitude of the node, and they can be solved analytically, as the equations are written in the form similar to those of pendulums with forced oscillations. However, for many of the asteroids the eccentricities and the inclinations are not so small, and, therefore, a new theory valid without any restriction should be formulated for secular perturbations. There are now several new theories which include more terms in the disturbing function, however, none of them are valid for secular resonant cases. In this paper only the author's theory(Kozai, 1962 and 1979) is taken up to explain the mechanisms mentioned above.

In order to reduce the degree of freedom for the equations of motion of the secular perturbations further, one must assume that all the disturbing planets move along circular orbits on the same plane. Then the potential becomes symmetrical with respect to the total angular momentum vector which is perpendicular to the orbital plane of the disturbing planets, and, therfore, the longitude of the ascending node disappears in the Hamiltonian if the common orbital plane of the planets which is called the invariable plane of the solar system is adopted as the reference plane.

In this paper the invariable plane is adopted as the reference plane. By this way most of the secular perturbation in the inclination derived by the classical linear theory disappears. Namely, the inclination to the invariable plane is more stable and nearly identical to the proper inclination in the classical theory.

Therefore, the $z$-component of the angular momentum of the asteroid, $\left(a\left(1-e^{2}\right)\right)^{1 / 2} \cos i$, becomes constant and the equations of motion are reduced to those of one degree of freedom with the variables, $G=\left(a\left(1-e^{2}\right)\right)^{1 / 2}$ and $g=$ the argument of perihelion. Also as $a$ is constant, $\Theta=\left(1-e^{2}\right)^{1 / 2} \cos i$ is constant and $X=G / a^{1 / 2}$ can be used as the variable replacing $G$.

Since the Hamiltonian is constant, one can derive $X$ as a function of $g$. Although one cannot express it by a simple known function, the variation of $X$ as a function of $g$ can be traced numerically by drawing equi-Hamiltonian-value curves on the $X-g$ plane. Since the Hamiltonian is a function symmetric with respect to $g=0^{\circ}$ and $180^{\circ}, g$ appears in the Hamiltonian as the combination of $2 g$ only. Therefore, the eccentricity and the inclination are expressed as periodic functions of $2 g$. 
When $\Theta$ is nearly equal to 1 , that is, both the eccentricity and the inclination are very small, they do no change so much as the possible maximum ranges of the variations for $X$ and $\cos i$ are between 1 and $\Theta$. As the value of $\Theta$ is reduced, the ranges of the variations of $X$ as well as the eccentricity and the inclination become wider. And $X$ as well as $i$ take their maximum values at $2 g=0^{\circ}$ and minimum values at $2 g=180^{\circ}$, and vice versa for the eccentricity. The argument of perihelion usually increases as time.

However, when the value of $\Theta$ is further reduced, say less than 0.8 , so-called libration region around the minimum value of the Hamiltonian can appear in the diagram on $X-2 g$ plane. And in the libration region the argument of perihelion does not make a complete revolution, but librates around $90^{\circ}$ or $270^{\circ}$ and the eccentricity and the inclination take their maximum and minimum values both at $2 g=180^{\circ}$. In the libration region an equilibrium point with the minimum value of the Hamiltonian exists.

\section{A Mechanism to Aroid Close Approach}

The Hamiltonian is the sum of the principal term and the disturbing function which is the sum of $m^{\prime} / \Delta$ over all the disturbing planets, where $m^{\prime}$ is the mass of the planet and $\Delta$ is the distance between the asteroid and the planet. Since the principal term which is a function of the semi-major axis only is constant, the value of the disturbing term, $C$, should be constant.

If only Jupiter is considered in the disturbing function as it is the principal disturbing planet, the value of $C$ is large when the asteroid can approach Jupiter very closely and it is small for the contrary case. As $C$ is constant, if the asteroid once approaches Jupiter it will approach it repeatedly and if the value of $C$ is small the asteroid has no chance to approach Jupiter very closely.

In Fig. 1 the equi- $C$-value curves for asteroids belonging to Pallas family ( $a$ $=2.77 \mathrm{AU}$ and $\Theta=0.8$ ) are shown with the points corresponding to the present values for the asteroids. In the figure the vertical coordinate represents the value of $X$ as well as $e$ and $i$ and the horizontal coordinate is $2 g$. And the figure shows how the values of $e$ and $i$ vary along one of the curves as functions of $2 g$. There is a libration region in the figure and 69 curves are drawn there with an equal interval of $C$. All the points of the asteroids of Pallas family are outside the libration region, however, very close to it. That is, their values of $C$ are very near its minimum value. Unless it is in the libration region, the value of $C$ is increased as the eccentricity is increased and, therefore, the inclination is decreased.

As the arguments of perihelion move, both the eccentricities and inclinations change very widely, when the values of $C$ are small, that is, the curves to be followed by such asteroids are in upper parts of the figure. Although the osculating eccentricities and inclinatins of the asteroids are scattered, respectively, between 0.1 and 0.5 and between $27^{\circ}$ and $38^{\circ}$, the figure shows that they have similar values of the proper elements. Therefore, it is believed that they belong to the same family, Pallas family. And it shows that even though the eccentricities are large they can avoid very close approach to Jupiter, because the aphelion distances are largest when the perihelia are far from the orbital plane of Jupiter $\left(2 g=180^{\circ}\right)$. When the 


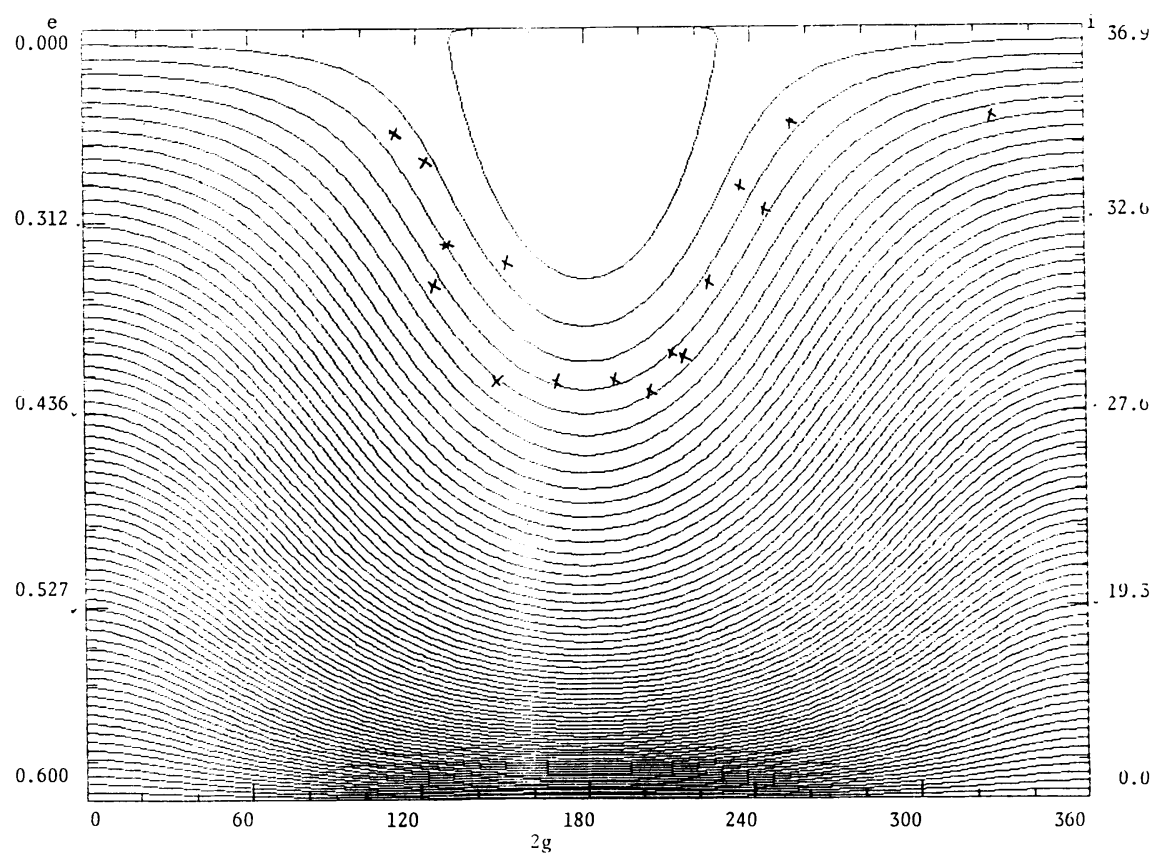

Fig. 1. Variations of eccentricities and inclinations for Pallas family asteroids.

aphelia on the major axes cross the orbital plane, the eccentricities and, therefore, the aphelion distances become small. This is a mechnism for such asteroids to avoid very close approach.

There are of nearly 5,000 numbered asteroids about 15 asteroids, for which the arguments of perielion librate. One of them is (1373) Cincinati, for which $a=3.41 \mathrm{AU}$, the eccentricity and the inclination vary, respectively, between 0.32 and 0.56 and between $29^{\circ}$ and $40^{\circ}$, and the argument of perihelion librates between $69^{\circ}$ and $111^{\circ}$. Therefore, the aphelion distance of this asteroid can extend up to 5.3AU, larger than the mean heliocentric radius of Jupiter. However, as the major axis of the orbit is very far from the orbital plane of Jupiter the closest distance to Jupiter cannot be less than 1.3AU, as the major axis of the asteroid cannot lie on the orbital plane of Jupiter. Certainly, it is another mechanism, by which asteroids with large eccentricities, namely, large heliocentric aphelion distances to avoid very close approach to Jupiter.

\section{A Criterion of Stabilty}

In Fig. 2 the values of $C$ in an arbitrary scale computed for all the numbered asteroids are plotted with respect to the semi-major axis. The size of the square depends on the value of $\Theta$. Namely, when the value of $\Theta$ is smaller, a larger size 


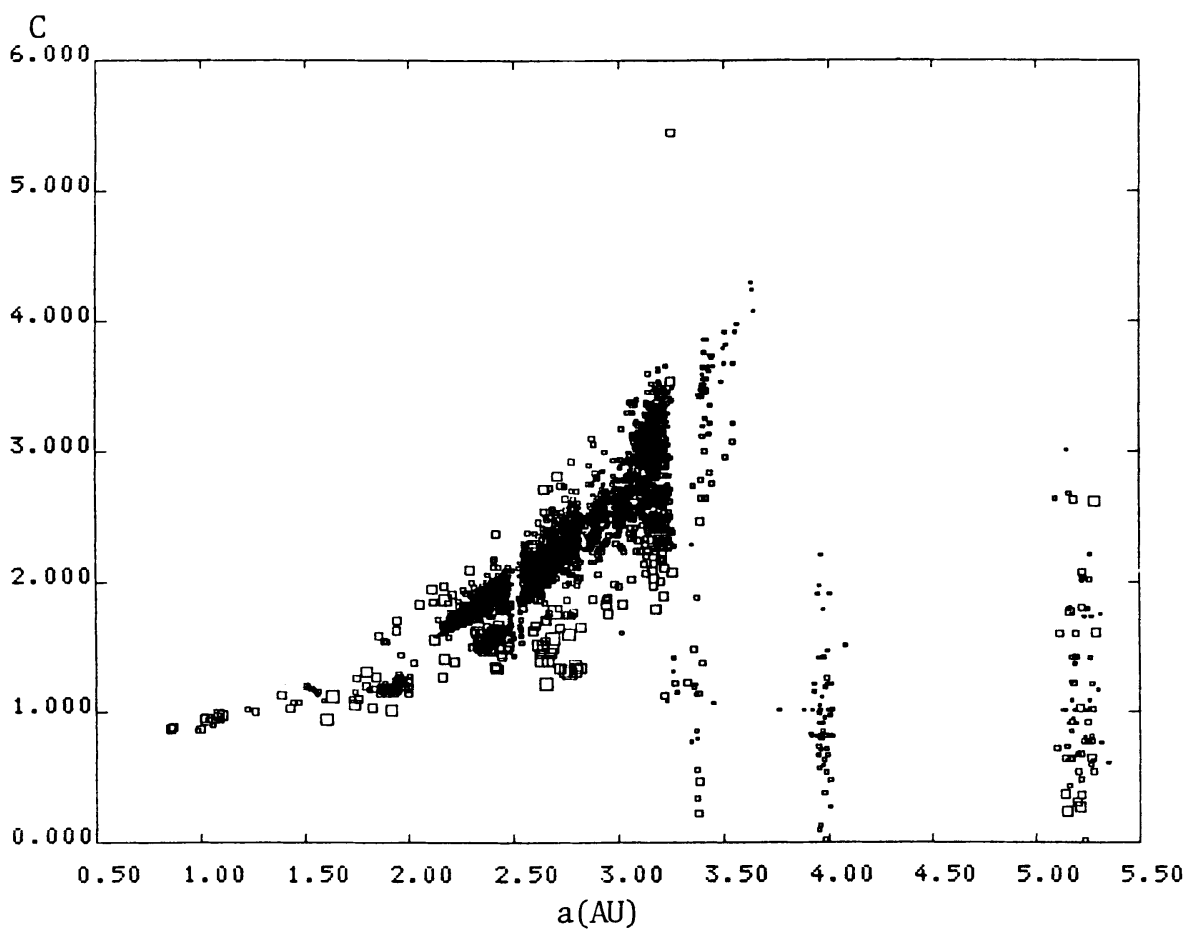

Fig. 2. The value of $C$ with respect to the semi-major axis.

is adopted. In Fig. 2, of course, Kirkwood gaps appear. And the value of $C$ is increased as the semi-major axis is increased, typically, for asteroids with small eccentricities and inclinations. However, when the semi-major axis is larger than 3.7AU, the value of $C$ does not increase because there only asteroids of Hilda, Thule and Trojan groups have been discovered.

For the asteroids belonging to the three groups it is assumed that the orbit of Jupiter is circular and the critical arguments are fixed at their stable equilibrium points to compute the values of $C$. However, it is not at all true that all the critical arguments do not move but stay at their equilibrium points, namely, for Hilda and Thule groups at $0^{\circ}$ and for Trojan group at $\pm 60^{\circ}$. That is why the values of $C$ for these asteroids in Fig. 2 are too low. However, it is expected that the differences from the real values, larger than those given here, are not enormous. Therefore, this figure seems to show that for most of the asteroids the values of $C$ are smaller than their respective averaged values.

There are several asteroids with larger values of $C$ and small values of $\Theta$. Such asteroids have large eccentricities and low inclinations and the eccentricities do no change so much as functions of the arguments of perihelion, as they follow curves in lower parts of diagrams similar to Fig. 1. Therefore, they often approach major 


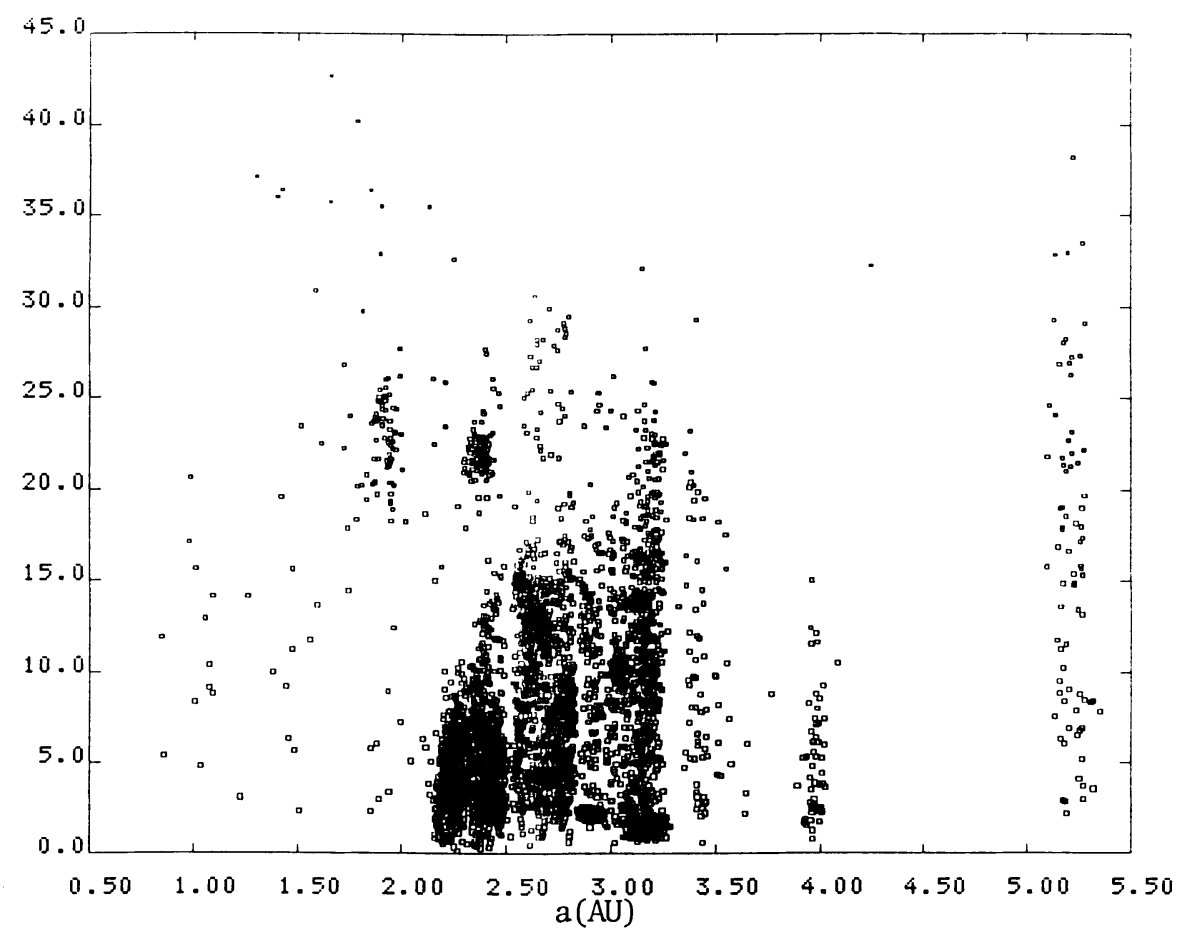

Fig. 3. $i-a$ diagram for asteroids.

planets, particularly, Jupiter very closely. In fact it is the dynamical characteristic of short-periodic comets, quite contrary to that of asteroids(Marsden, 1970). Therefore, it seems to the author that such asteroids are dynamically of comet-type and have possibility that they are distinct comets.

However, for asteroids which have smaller $\Theta$, the values of $C$ are smaller than those for the asteroids with small eccentricities and inclinations. It is a typical characteristic of asteroid orbits and is a reason why the asteroid motions are stable.

\section{Discussions}

In Fig. 2 a group of asteroids near $a=2.3 \sim 2.5 \mathrm{AU}$ which have lower values of $C$ belong to Phocaea family with $\Theta=0.86 \sim 0.91$ and $i=20^{\circ} \sim 23^{\circ}$ and that near $a=2.7 \sim 2.8 \mathrm{AU}$ and with lower values of $\Theta$ and $C$ belong to Pallas families, for which the values of $\Theta$ are nearly 0.8 . That is, even though they have rather larger values of the eccentricities they have no chance to approach Jupiter very closely, as they follow curves in upper parts of the diagrams similar to Fig. 1.

In Fig. $3 i-a$ diagram for the numbered asteroids is shown and there several 
$\mathrm{e}$

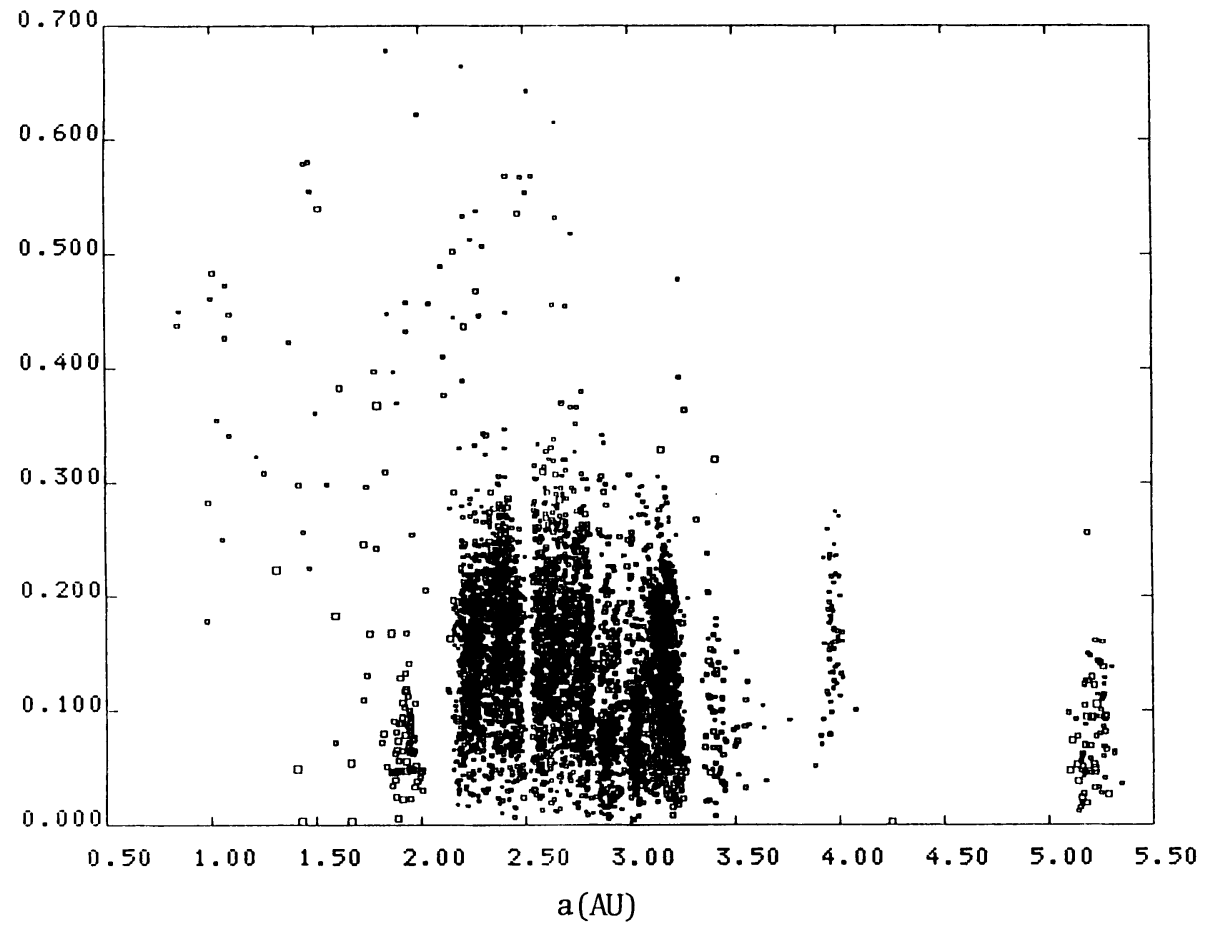

Fig. 4. $e-a$ diagram for asteroids.

clusterings corresponding to families are recognized. In fact there are several major families, for which member asteroids have similar values of the semi-major axes, and two of the stable proper elements, $\Theta$ and the minimum value of the orbital inclination, $i_{\min }$. In fact the inclination in Fig. 2 is this minimum value as the function of the argument of perihelion.

And in Fig. $4 e-a$ diagram for the numbered asteroids are shown. Here even for the families their values are scattered partly because some of the secular perturbations are not taken into account and they are really in wider range than the inclination. It is remarkable to find in Figs. 3 and 4 that Trojan asteroids usually high inclinations and low eccentricities, both contributing to avoid very close approach to Jupiter.

For families one can notice that most of them are bounded in Figs. 3 and 4 at least partly by resonance lines, either mean motions and/or secular motions. This means that the families could not be extended over any Kirkwood and secular motion gap. Also it is true that it is difficult to find out any asteroid with large eccentricity and low inclination nearby, which could sweep up asteroids to make any of the gaps. And there are many evidences that the families, at least most of them, 
were created by collisions among asteroids, and, therefore, many small fragmented asteroids are found in the families.

It can be also recognized that near Kirkwood gaps only faint asteroids are found. In fact when the number distribution histogram of bright asteroids only with respect to their semi-major axes is drawn, the gaps become more clear, namely, they are indeed gaps. This may show that the gaps may have been created by collisions as there is no other plausible explanation about it.

It is true that for almost all of the asteroids the motions are stable, because it seems to the author that some dynamical evolution processes have made them stable. Still it is very difficult to find out exactly what have taken place to make them stable.

\section{References}

Kozai, Y. 1962. Secular Perturbations of Asteroids with High Inclinations and Eccentricities, Astron. J., 67, 59l-598.

Kozai, Y. 1979. Secular Perturbations of Asteroids and Comets, In Dynamics of the Solar System, ed. by R.L.Duncombe, 231-237, Reidel Publ. Co.

Marsden, B.G. 1970. On the Relationship between Comets and Minor Planets, Astron. J., 75, 206-217.

\section{Discussion}

S.F.Dermott - That asteroids are smaller than average near the Kirkwood gaps was noted by E.Bowell about 10 years ago. I investigated this with Carl Murray (Nature, $290,664,1981)$. We showed that this is probably due to observational selection associated with the fact that small asteroids tend to have small inclinations. The shape of the resonance regions in a-I space is such that asteroids near a Kirkwood gap tend to have small inclinations. This is true for both the bright and faint asteroids, but only the faint asteroids show the apparent mass variation near the gaps.

Y.Kozai - Near 2:1 gap there are several asteroids with high inclination.

A.Morbidelli - In your work (1969) you have computed that a region of $\omega$-libration appears at about $30^{\circ}$ of inclination in the asteroid belt. Have you ever extended these computations in the region outside Jupiter?

Y.Kozai - Yes. I applied my method to Pluto-Neptune case as well as short-periodic comets and Hilda, for which the perihelion distance will become $2 \mathrm{AU}$ in future. 